\title{
Evaluation of Elite Bivoltine Silkworm (Bombyx mori L.) Foundation Crosses Suitable for Temperate Region of Jammu \& Kashmir
}

\author{
Bharath Kumar Neelaboina*, Shivkumar, Mir Nisar Ahmad and M.K. Ghosh
}

Silkworm Breeding and Genetics Section, Central Sericultural Research and Training Institute, Central Silk Board, Pampore, Jammu and Kashmir, India

*Corresponding author

\begin{tabular}{|l|}
\hline Ke y w o r d s \\
Silkworm, \\
$\begin{array}{l}\text { Foundation cross, } \\
\text { Temperate, } \\
\text { Evaluation index }\end{array}$ \\
\hline Article Info \\
\hline $\begin{array}{l}\text { Accepted: } \\
\text { 20 December2018 } \\
\text { Available Online: } \\
\text { 10 January } 2019\end{array}$ \\
\hline
\end{tabular}

A B S T R A C T
The present investigation was carried out at Central Sericultural Research and Training Institute, Pampore, Jammu \& Kashmir (J\&K) during spring, summer and autumn, 2018 to study the performance of 24 bivoltine silkworm, Bombyx mori L, foundation crosses (12 Oval \& 12 Constricted) under temperate climatic conditions. The rearing was carried out under uniformed laboratory condition by adopting standard method. These 24 bivoltine silkworm foundation crosses (FC) were evaluated for their performance in eight metric traits viz., fecundity (No.), hatching (\%), yield per 10,000 larvae by number and by weight $(\mathrm{kg})$, single cocoon weight $(\mathrm{g})$, single shell weight $(\mathrm{g})$, shell ratio $(\%)$ and pupation rate $(\%)$. The performance of foundation crosses varied from season to season since the parental races involved in these foundation crosses have different origin with specific characteristics. The data generated was analyzed statistically and subjected to multiple trait Evaluation index. On the basis of the evaluation index (E.I) values ranking constricted FC's viz., SK-6 $\times$ SK-7, Pam-117 $\times$ APS-4 and Pam-117 $\times$ SK-7 performed well in all the three seasons with E.I above 50 and SK-7 $\times$ Pam-117 performed well in autumn season only. In case of Oval FC's viz., CSR-27 $\times$ Pam-114 \& CSR-50 $\times$ Pam-114 along with their reciprocals performed well and recorded E.I above 50 in all the three seasons whereas CSR-27 $\times$ CSR-50 \& APS-5 $\times$ Pam-114 recorded E.I above 50 in spring and summer only. CSR-50 $\times$ APS-5 and its reciprocal were found superior in performance only in autumn season. Based on their performance in all the three seasons, these foundation crosses will be utilized for the development of bivoltine silkworm double hybrid suitable to temperate region of J\&K as an alternate for ruling CSR double hybrid.

\section{Introduction}

Mulberry belonging to family Moraceae is primarily cultivated for rearing of silkworms and its contribution towards successful rearing is $38.2 \%$ (Miyashita, 1986). The common silkworm Bombyx mori Linnaeus (Lepidoptera: Bombycidae) spins valuable silk fibre, making it one of the most beneficial insect to mankind and is becoming an attractive multifunctional material for both textile and non textile uses Tsukada (2005). The practice of sericulture consist of two major activities viz., cultivation of mulberry plants for producing healthy leaf for feeding to the silkworm larvae and the rearing of 
silkworm larvae to produce the quality cocoons, which is the raw material for the silk reeling industry (Bharath Kumar et al., 2017; Bharath Kumar et al., 2018a). With the reorientation of silkworm breeding approaches aimed at sustainability and increased qualitative silk production, unstinted and coordinated efforts by various silkworm breeders in the country (Basavaraja et al., 1995) resulted in the development of many bivoltine silkworm breeds and hybrids over the last few decades. Till date limited number of silkworm breeds / hybrids has been developed for the spring season under temperate climatic conditions of Kashmir (Trag et al., 1992) Whereas, during summer 10-15 percent farmers take up sericulture in temperate regions of Kashmir (Sahaf et al., 2016) few attempts have also been made for identification of hybrids for summer or autumn season (Farooq et al., 2006; Malik et al., 2009 and Nisar et al., 2013). It is also need of hour to develop silkworm breeds/ hybrids withstanding the climate change (Bharath Kumar et al., 2018b).

In a single hybrid, hybrid vigour between two genetically distant parents has the potential for better productivity, but the double hybrids involving four parental breeds of diverse characteristics (disease tolerance, high productivity etc.) ensure the sustainability and better financial returns to the farmer. The flexibility in genetic constitution within the population and polygenic expression of several economic characteristics in silkworm double hybrids is more stable than the single hybrids under sub-optimal conditions. The temperate conditions of Jammu \& Kashmir demand the silkworm breeds/hybrids which possess disease tolerance coupled with high productivity. The bivoltine double hybrids have better genetic plasticity to buffer against adverse climatic conditions resulting in crop stability (Basavaraja et al., 2006). The development of sustainable silkworm double hybrids characterized for disease tolerance and high productivity would provide better opportunities for the up liftment of socio economic conditions of farmers in North West India

Hence, the present study aims to evaluate the performance of 24 foundation crosses in all the three seasons at CSR\&TI, Pampore to develop the bivoltine silkworm double hybrid suitable for Kashmir valley.

\section{Materials and Methods}

Twenty four bivoltine silkworm foundation crosses (12 Oval and 12 Constricted) were reared during spring (May-June), summer (July-August) and autumn (AugustSeptember), 2018 at Central Sericultural Research and Training Institute, Central Silk Board, Pampore, Kashmir. The standard rearing techniques were followed Krishnaswamy (1978). The important quantitative and qualitative traits viz., fecundity, hatching percentage, yield per 10,000 larvae by weight, single cocoon weight, single shell weight, shell ratio and pupation rate were recorded in all the 24 bivoltine silkworm foundation crosses during spring, summer and autumn, 2018. All the foundation crosses were reared following completely randomised design with three replications each and 250 larvae were maintained in each replication after $3^{\text {rd }}$ moult. At the end of $5^{\text {th }}$ instar, the spinning larvae were collected manually and mounted in plastic collapsible mountages. The evaluation index value was calculated for all the eight traits studied. The evaluation index (EI) was calculated as per the below mentioned procedure (Mano et al., 1993).

Evaluation Index $=\frac{\mathrm{A}-\mathrm{B}}{\mathrm{C}} \times 10+50$

Where, $\mathrm{A}=$ Value obtained for a particular trait in a particular breed 
$\mathrm{B}=$ Mean value of a particular trait of all the breeds

$\mathrm{C}=$ Standard deviation of a particular trait of all the breeds

$10=$ Standard unit

$50=$ Fixed value

The index value obtained for all the traits was combined and the average EI values were obtained. The EI value fixed for the selection of a line is 50 or $>50$. The line, which scored above the limit, is considered to possess greater economic value.

\section{Results and Discussion}

The present research findings revealed that six constricted foundation crosses viz., APS-4 $\times$ SK-7, SK-6 × APS-4, SK-6 × Pam-117, SK-6 $\times$ SK-7, Pam-117 $\times$ APS-4 and Pam-117 $\times$ SK-7 performed well over the control FC1 (CSR6 $\times$ CSR26) in the spring season (Table 1) with E.I values 51.57, 50.31, 52.94, 55.41, 55.77 and 61.32 (Table 2) respectively along with six oval foundation crosses viz., CSR-27 $\times$ CSR-50 (55.37), CSR-27 × Pam-114 (54.93), CSR-50 × Pam-114 (52.91), Pam-114 $\times$ CSR-27 (56.26), Pam-114 × CSR-50 (56.60) and APS-5 $\times$ Pam-114 (53.65) (Table 3 and 4). Among the constricted foundation crosses SK-6 $\times$ SK-7, Pam-117 $\times$ APS-4 and Pam-117 $\times$ SK-7 performed well in the summer season with E.I values 59.13, 58.72, and 59.66 (Table 5 and 6) whereas in case of oval foundation crosses CSR-27 $\times$ CSR-50 (51.15), CSR-27 $\times$ Pam-114 (53.18), CSR-50 × Pam-114 (55.38), Pam-114 × CSR-27 (57.50), Pam-114 × CSR50 (56.51) and APS-5 $\times$ Pam-114 (56.44) performed well over the control FC2 (CSR2 $\times$ CSR27) in summer 2018 (Table 7 and 8). In autumn season SK-6 $\times$ SK-7, Pam-117 $\times$ APS-4, Pam-117 $\times$ SK-7 and SK-7 $\times$ Pam117 excelled over the other constricted foundation crosses with E.I values 57.34, 55.55, 60.82 and 53.40 respectively (Table 9 and 10) along with oval foundation crosses viz., CSR-27 × Pam-114 (53.33), CSR-50 × Pam-114 (61.72), CSR-50 × APS-5 (52.46), Pam-114 × CSR-27 (61.62), Pam-114 × CSR50 (54.04) and APS-5 $\times$ CSR-50 (53.95) (Table 11 and 12).

Three constricted FC viz., SK-6 $\times$ SK-7, Pam$117 \times$ APS-4 and Pam-117 $\times$ SK-7 performed well in spring, summer and autumn, 2018 whereas APS-4 $\times$ SK-7, SK-6 $\times$ APS-4 and SK-6 $\times$ Pam-117 observed with E.I values above 50 only in spring season. Among the constricted foundation crosses SK-7 $\times$ Pam117 excelled in only autumn season, 2018. In case of oval foundation crosses CSR-27 $\times$ Pam-114, CSR-50 × Pam-114, Pam-114 $\times$ CSR-27 and Pam-114 $\times$ CSR-50 performed well in spring, summer and autumn, 2018 whereas CSR-27 $\times$ CSR-50 and APS-5 $\times$ Pam-114 recorded E.I values above 50 in spring and summer, 2018. Among the oval foundation crosses CSR-50 $\times$ APS-5 and its reciprocal performed well in autumn only. Similar studies were conducted by Moorthy et al., (2011) for identification of suitable bivoltine foundation cross for sustainable silkworm seed crop in tropics and found that D6 (P) N x SK4C shown higher cocoon yield of $12.8 \mathrm{~kg} / 10000$ larvae with $91 \%$ pupation compared to $9.8 \mathrm{~kg}$ cocoon yield $/ 10000$ larvae with $67 \%$ pupation in control, NB18 x P5.

Success of any breeding programme is dependent on the degree of genetic variability of the available genetic resources and the selection of suitable and genetically diverse parental breeds to build heterotic combinations. Higher the variability more is the scope for obtaining higher amount of heterotic potential and also more variability is expected to surface in segregating generations of such crosses (Shabir Ahmad Bhat et al., 2018). Similar studies based on evaluation index values had also been conducted by Quadir et al., (2000), Suresh Kumar et al., (2006) and Nisar et al., (2013). 
Table.1 Rearing performance of the constricted foundation crosses during spring, 2018

\begin{tabular}{|c|c|c|c|c|c|c|c|c|}
\hline \multirow[t]{2}{*}{ Parental Breeds } & \multirow{2}{*}{$\begin{array}{l}\text { Fecundity } \\
\text { (No.) }\end{array}$} & \multirow{2}{*}{$\begin{array}{l}\text { Hatching } \\
(\%)\end{array}$} & \multicolumn{2}{|c|}{ Yield / 10, 000 larvae brushed } & \multirow{2}{*}{$\begin{array}{l}\text { Single cocoon } \\
\text { wt. (g) }\end{array}$} & \multirow{2}{*}{$\begin{array}{l}\text { Single shell } \\
\text { wt.(g) }\end{array}$} & \multirow{2}{*}{$\begin{array}{c}\text { Shell ratio } \\
(\%)\end{array}$} & \multirow{2}{*}{$\begin{array}{l}\text { Pupation rate } \\
(\%)\end{array}$} \\
\hline & & & By No. & By Wt.(kg) & & & & \\
\hline APS-4 $\times$ SK-6 & 521 & 95.67 & 9580 & 13.4 & 1.57 & 0.31 & 19.75 & 88.00 \\
\hline APS-4 × Pam-117 & 532 & 96.44 & 9720 & 12.6 & 1.46 & 0.28 & 19.18 & 89.00 \\
\hline APS-4 × SK-7 & 554 & 96.26 & 9480 & 14.7 & 1.72 & 0.31 & 18.02 & 90.00 \\
\hline SK-6 × APS-4 & 570 & 96.08 & 9560 & 13.3 & 1.56 & 0.31 & 19.87 & 89.00 \\
\hline SK-6 $\times$ Pam-117 & 543 & 96.72 & 9600 & 14.6 & 1.69 & 0.32 & 18.93 & 88.00 \\
\hline SK-6 $\times$ SK-7 & 541 & 96.88 & 9680 & 13.7 & 1.59 & 0.32 & 20.13 & 92.00 \\
\hline Pam-117 $\times$ APS-4 & 501 & 95.21 & 9700 & 14.5 & 1.66 & 0.34 & 20.48 & 92.00 \\
\hline Pam-117 × SK-6 & 574 & 95.16 & 9480 & 12.7 & 1.51 & 0.29 & 19.21 & 89.00 \\
\hline Pam-117 × SK-7 & 565 & 94.55 & 9600 & 15.4 & 1.77 & 0.38 & 21.47 & 91.00 \\
\hline SK-7 $\times$ APS- 4 & 559 & 95.03 & 9360 & 13.8 & 1.64 & 0.30 & 18.29 & 88.00 \\
\hline SK-7 × SK-6 & 515 & 94.97 & 9540 & 13.1 & 1.54 & 0.30 & 19.48 & 91.00 \\
\hline SK-7 $\times$ Pam-117 & 475 & 92.75 & 9600 & 13.5 & 1.57 & 0.30 & 19.11 & 92.00 \\
\hline FC-1 & 449 & 95.50 & 9387 & 13.63 & 1.62 & 0.33 & 20.37 & 93.00 \\
\hline Average & 531 & 95.48 & 9561 & 13.75 & 1.61 & 0.31 & 19.56 & 90.15 \\
\hline $\mathrm{SD}$ & 38 & 1.09 & 111 & 0.82 & 0.09 & 0.03 & 0.93 & 1.77 \\
\hline
\end{tabular}

Table.2 Evaluation index values of the constricted foundation crosses during spring, 2018

\begin{tabular}{|c|c|c|c|c|c|c|c|c|c|}
\hline \multirow[t]{2}{*}{ Parental breeds } & \multirow{2}{*}{$\begin{array}{l}\text { Fecundity } \\
\text { (No.) }\end{array}$} & \multirow{2}{*}{$\begin{array}{c}\text { Hatching } \\
(\%)\end{array}$} & \multicolumn{2}{|c|}{ Yield / 10, 000 larvae brushed } & \multirow{2}{*}{$\begin{array}{c}\text { Single cocoon } \\
\text { wt. (g) }\end{array}$} & \multirow{2}{*}{$\begin{array}{l}\text { Single shell } \\
\text { wt.(g) }\end{array}$} & \multirow{2}{*}{$\begin{array}{c}\text { Shell ratio } \\
(\%)\end{array}$} & \multirow{2}{*}{$\begin{array}{c}\text { Pupation rate } \\
(\%)\end{array}$} & \multirow{2}{*}{$\begin{array}{l}\text { Average } \\
\text { E.I }\end{array}$} \\
\hline & & & By No. & By Wt.(kg) & & & & & \\
\hline APS-4 $\times$ SK-6 & 47.37 & 51.74 & 51.71 & 46.22 & 45.56 & 50.00 & 51.99 & 40.00 & 48.07 \\
\hline APS-4 $\times$ Pam-117 & 50.26 & 58.81 & 64.32 & 35.85 & 33.33 & 40.00 & 45.89 & 45.00 & 46.68 \\
\hline APS $-4 \times$ SK-7 & 56.05 & 57.16 & 42.70 & 60.98 & 62.22 & 50.00 & 33.48 & 50.00 & 51.57 \\
\hline SK-6 $\times$ APS-4 & 60.26 & 55.50 & 49.91 & 44.02 & 44.44 & 50.00 & 53.35 & 45.00 & 50.31 \\
\hline SK-6 × Pam-117 & 53.16 & 61.38 & 53.51 & 60.00 & 58.89 & 53.33 & 43.28 & 40.00 & 52.94 \\
\hline SK-6 $\times$ SK-7 & 52.63 & 62.84 & 60.72 & 49.88 & 47.78 & 53.33 & 56.08 & 60.00 & 55.41 \\
\hline Pam-117 $\times$ APS-4 & 42.11 & 47.52 & 62.52 & 58.54 & 55.56 & 60.00 & 59.91 & 60.00 & 55.77 \\
\hline Pam-117 × SK-6 & 61.32 & 47.06 & 42.70 & 36.79 & 38.89 & 43.33 & 46.19 & 45.00 & 45.16 \\
\hline Pam-117 × SK-7 & 58.95 & 41.47 & 53.51 & 70.02 & 67.78 & 73.33 & 70.53 & 55.00 & 61.32 \\
\hline SK-7 $\times$ APS-4 & 57.37 & 45.87 & 31.89 & 50.00 & 53.33 & 46.67 & 36.37 & 40.00 & 45.19 \\
\hline SK-7 × SK-6 & 45.79 & 45.32 & 48.11 & 41.98 & 42.22 & 46.67 & 49.15 & 55.00 & 46.78 \\
\hline SK-7 $\times$ Pam-117 & 35.26 & 24.95 & 53.51 & 46.61 & 45.56 & 46.67 & 45.14 & 60.00 & 44.71 \\
\hline FC-1 & 28.42 & 50.18 & 34.32 & 48.63 & 51.11 & 56.67 & 58.71 & 65.00 & 49.13 \\
\hline
\end{tabular}


Table.3 Rearing Performance of the oval foundation crosses during spring, 2018

\begin{tabular}{|c|c|c|c|c|c|c|c|c|}
\hline \multirow[t]{2}{*}{ Parental breeds } & \multirow{2}{*}{$\begin{array}{l}\text { Fecundity } \\
\text { (No.) }\end{array}$} & \multirow{2}{*}{$\begin{array}{l}\text { Hatching } \\
(\%)\end{array}$} & \multicolumn{2}{|c|}{ Yield / 10, 000 larvae brushed } & \multirow{2}{*}{$\begin{array}{c}\text { Single cocoon } \\
\text { wt. (g) }\end{array}$} & \multirow{2}{*}{$\begin{array}{l}\text { Single shell } \\
\text { wt.(g) }\end{array}$} & \multirow{2}{*}{$\begin{array}{c}\text { Shell ratio } \\
(\%)\end{array}$} & \multirow{2}{*}{$\begin{array}{c}\text { Pupation rate } \\
(\%)\end{array}$} \\
\hline & & & By No. & By Wt.(kg) & & & & \\
\hline CSR-27 $\times$ CSR -50 & 492 & 94.30 & 9700 & 15.04 & 1.72 & 0.37 & 21.51 & 94.00 \\
\hline CSR-27 $\times$ Pam-114 & 487 & 94.63 & 9780 & 15.12 & 1.71 & 0.36 & 21.05 & 94.00 \\
\hline CSR-27 × APS-5 & 476 & 88.02 & 9620 & 14.03 & 1.63 & 0.34 & 20.86 & 91.00 \\
\hline CSR-50 $\times$ CSR -27 & 479 & 91.23 & 9740 & 14.23 & 1.63 & 0.34 & 20.86 & 95.00 \\
\hline CSR-50 $\times$ Pam-114 & 510 & 92.32 & 9660 & 15.11 & 1.73 & 0.37 & 21.39 & 92.00 \\
\hline $\mathrm{CSR}-50 \times \mathrm{APS}-5$ & 578 & 97.25 & 9500 & 13.93 & 1.64 & 0.34 & 20.73 & 91.00 \\
\hline Pam-114 × CSR-27 & 569 & 94.22 & 9680 & 15.29 & 1.75 & 0.37 & 21.14 & 92.00 \\
\hline Pam-114 × CSR-50 & 524 & 93.12 & 9600 & 15.87 & 1.82 & 0.38 & 20.88 & 93.00 \\
\hline Pam-114 × APS-5 & 535 & 94.54 & 9480 & 13.57 & 1.60 & 0.30 & 18.75 & 90.00 \\
\hline APS- $5 \times$ CSR -27 & 512 & 96.24 & 9440 & 14.50 & 1.71 & 0.34 & 19.88 & 90.00 \\
\hline APS- $5 \times$ CSR -50 & 526 & 93.30 & 9600 & 14.48 & 1.68 & 0.34 & 20.24 & 92.00 \\
\hline APS-5 $\times$ Pam-114 & 561 & 94.24 & 9640 & 15.61 & 1.79 & 0.36 & 20.11 & 91.00 \\
\hline $\mathrm{FC} 2$ & 597 & 96.27 & 9507 & 15.07 & 1.75 & 0.36 & 20.72 & 92.00 \\
\hline Average & 527 & 93.82 & 9611 & 14.76 & 1.70 & 0.35 & 20.63 & 92.08 \\
\hline SD & 40 & 2.39 & 104 & 0.69 & 0.07 & 0.02 & 0.74 & 1.55 \\
\hline
\end{tabular}

Table.4 Evaluation index values of the oval foundation crosses during spring, 2018

\begin{tabular}{|c|c|c|c|c|c|c|c|c|c|}
\hline \multirow[t]{2}{*}{ Parental breeds } & \multirow{2}{*}{$\begin{array}{l}\text { Fecundity } \\
\text { (No.) }\end{array}$} & \multirow{2}{*}{$\begin{array}{l}\text { Hatching } \\
(\%)\end{array}$} & \multicolumn{2}{|c|}{ Yield / 10, 000 larvae brushed } & \multirow{2}{*}{$\begin{array}{c}\text { Single cocoon } \\
\text { wt. }(\mathrm{g})\end{array}$} & \multirow{2}{*}{$\begin{array}{c}\text { Single shell } \\
\text { wt.(g) }\end{array}$} & \multirow{2}{*}{$\begin{array}{c}\text { Shell ratio } \\
(\%)\end{array}$} & \multirow{2}{*}{$\begin{array}{c}\text { Pupation rate } \\
(\%)\end{array}$} & \multirow{2}{*}{$\begin{array}{c}\text { Average } \\
\text { E.I }\end{array}$} \\
\hline & & & By No. & By Wt.(kg) & & & & & \\
\hline CSR-27 $\times$ CSR -50 & 41.25 & 52.01 & 58.56 & 54.00 & 52.86 & 60.00 & 61.91 & 62.39 & 55.37 \\
\hline CSR-27 × Pam-114 & 40.00 & 53.39 & 66.25 & 55.28 & 51.43 & 55.00 & 55.71 & 62.39 & 54.93 \\
\hline CSR-27 × APS-5 & 37.25 & 25.73 & 50.87 & 39.46 & 40.00 & 45.00 & 53.09 & 43.03 & 41.80 \\
\hline CSR-50 $\times$ CSR-27 & 38.00 & 39.16 & 62.40 & 42.29 & 40.00 & 45.00 & 53.09 & 68.84 & 48.60 \\
\hline CSR-50 $\times$ Pam-114 & 45.75 & 43.72 & 54.71 & 55.10 & 54.29 & 60.00 & 60.23 & 49.48 & 52.91 \\
\hline CSR $-50 \times$ APS -5 & 62.75 & 64.35 & 39.33 & 38.01 & 41.43 & 45.00 & 51.37 & 43.03 & 48.16 \\
\hline Pam-114 × CSR-27 & 60.50 & 51.67 & 56.63 & 57.71 & 57.14 & 60.00 & 56.93 & 49.48 & 56.26 \\
\hline Pam-114 × CSR-50 & 49.25 & 47.07 & 48.94 & 66.12 & 67.14 & 65.00 & 53.37 & 55.94 & 56.60 \\
\hline Pam-114 × APS-5 & 52.00 & 53.01 & 37.40 & 32.72 & 35.71 & 25.00 & 24.59 & 36.58 & 37.13 \\
\hline APS-5 × CSR-27 & 46.25 & 60.13 & 33.56 & 46.16 & 51.43 & 45.00 & 39.91 & 36.58 & 44.88 \\
\hline APS-5 × CSR-50 & 49.75 & 47.82 & 48.94 & 45.94 & 47.14 & 45.00 & 44.70 & 49.48 & 47.35 \\
\hline APS-5 × Pam-114 & 58.50 & 51.76 & 52.79 & 62.28 & 62.86 & 55.00 & 43.00 & 43.03 & 53.65 \\
\hline FC2 & 67.50 & 60.25 & 40.00 & 54.46 & 57.14 & 55.00 & 51.22 & 49.48 & 54.38 \\
\hline
\end{tabular}


Table.5 Rearing Performance of the constricted foundation crosses during summer, 2018

\begin{tabular}{|c|c|c|c|c|c|c|c|c|}
\hline \multirow[t]{2}{*}{ Parental breeds } & \multirow{2}{*}{$\begin{array}{l}\text { Fecundity } \\
\text { (No.) }\end{array}$} & \multirow{2}{*}{$\begin{array}{c}\text { Hatching } \\
(\%)\end{array}$} & \multicolumn{2}{|c|}{ Yield / 10, 000 larvae brushed } & \multirow{2}{*}{$\begin{array}{l}\text { Single cocoon } \\
\text { wt. (g) }\end{array}$} & \multirow{2}{*}{$\begin{array}{l}\text { Single shell } \\
\text { wt.(g) }\end{array}$} & \multirow{2}{*}{$\begin{array}{c}\text { Shell ratio } \\
(\%)\end{array}$} & \multirow{2}{*}{$\begin{array}{c}\text { Pupation rate } \\
(\%)\end{array}$} \\
\hline & & & By No. & By Wt.(kg) & & & & \\
\hline APS-4 $\times$ SK-6 & 450 & 91.08 & 9480 & 14.32 & 1.70 & 0.34 & 19.71 & 93.00 \\
\hline APS-4 $\times$ Pam-117 & 517 & 92.66 & 9520 & 13.38 & 1.60 & 0.31 & 19.12 & 92.50 \\
\hline APS-4 × SK-7 & 544 & 92.21 & 9600 & 14.37 & 1.69 & 0.32 & 18.99 & 92.00 \\
\hline SK-6 $\times$ APS-4 & 552 & 92.28 & 9520 & 14.77 & 1.74 & 0.32 & 18.39 & 93.00 \\
\hline SK-6 $\times$ Pam-117 & 517 & 91.56 & 9640 & 13.77 & 1.62 & 0.32 & 19.85 & 94.50 \\
\hline SK-6 $\times$ SK-7 & 561 & 95.14 & 9660 & 13.94 & 1.63 & 0.34 & 20.86 & 95.50 \\
\hline Pam-117 $\times$ APS-4 & 559 & 95.07 & 9600 & 14.57 & 1.71 & 0.35 & 20.53 & 92.50 \\
\hline Pam-117 × SK-6 & 517 & 92.09 & 9540 & 13.80 & 1.64 & 0.33 & 19.88 & 94.00 \\
\hline Pam-117 × SK-7 & 587 & 94.26 & 9620 & 14.31 & 1.68 & 0.35 & 20.90 & 93.50 \\
\hline SK-7 $\times$ APS-4 & 539 & 94.88 & 9500 & 13.84 & 1.65 & 0.32 & 19.47 & 92.50 \\
\hline SK-7 × SK-6 & 535 & 94.27 & 9500 & 14.59 & 1.73 & 0.32 & 18.55 & 92.00 \\
\hline SK-7 $\times$ Pam-117 & 509 & 92.28 & 9480 & 14.45 & 1.72 & 0.33 & 19.23 & 92.50 \\
\hline FC-1 & 543 & 93.21 & 9600 & 14.28 & 1.68 & 0.35 & 20.60 & 93.00 \\
\hline Average & 533 & 93.15 & 9558 & 14.18 & 1.67 & 0.33 & 19.70 & 93.12 \\
\hline SD & 33.26 & 1.41 & 63.49 & 0.40 & 0.05 & 0.01 & 0.84 & 1.02 \\
\hline
\end{tabular}

Table.6 Evaluation index values of the constricted foundation crosses during summer, 2018

\begin{tabular}{|c|c|c|c|c|c|c|c|c|c|}
\hline \multirow[t]{2}{*}{ Parental breeds } & \multirow{2}{*}{$\begin{array}{l}\text { Fecundity } \\
\text { (No.) }\end{array}$} & \multirow{2}{*}{$\begin{array}{c}\text { Hatching } \\
(\%)\end{array}$} & \multicolumn{2}{|c|}{ Yield / 10, 000 larvae brushed } & \multirow{2}{*}{$\begin{array}{c}\text { Single cocoon } \\
\text { wt. (g) }\end{array}$} & \multirow{2}{*}{$\begin{array}{c}\text { Single shell } \\
\text { wt.(g) }\end{array}$} & \multirow{2}{*}{$\begin{array}{c}\text { Shell ratio } \\
(\%)\end{array}$} & \multirow{2}{*}{$\begin{array}{c}\text { Pupation rate } \\
(\%)\end{array}$} & \multirow{2}{*}{$\begin{array}{c}\text { Average } \\
\text { E.I }\end{array}$} \\
\hline & & & By No. & By Wt.(kg) & & & & & \\
\hline APS-4 $\times$ SK-6 & 24.95 & 35.32 & 37.71 & 53.38 & 56.00 & 55.00 & 50.06 & 50.00 & 45.30 \\
\hline APS-4 $\times$ Pam-117 & 45.13 & 46.52 & 44.01 & 30.04 & 35.00 & 25.00 & 43.15 & 45.10 & 39.24 \\
\hline APS-4 × SK-7 & 53.31 & 43.33 & 56.62 & 54.87 & 53.00 & 40.00 & 41.51 & 40.20 & 47.85 \\
\hline SK-6 $\times$ APS-4 & 55.59 & 43.79 & 44.01 & 64.68 & 64.00 & 40.00 & 34.44 & 50.00 & 49.57 \\
\hline SK-6 $\times$ Pam-117 & 45.16 & 38.69 & 62.92 & 39.78 & 39.00 & 40.00 & 51.74 & 64.71 & 47.75 \\
\hline SK-6 $\times$ SK-7 & 58.42 & 64.11 & 66.07 & 44.12 & 42.00 & 60.00 & 63.83 & 74.51 & 59.13 \\
\hline Pam-117 × APS-4 & 57.88 & 63.62 & 56.62 & 59.67 & 57.00 & 70.00 & 59.87 & 45.10 & 58.72 \\
\hline Pam-117 × SK-6 & 45.10 & 42.48 & 47.16 & 40.52 & 43.00 & 45.00 & 52.19 & 59.80 & 46.91 \\
\hline Pam-117 × SK-7 & 66.18 & 57.84 & 59.77 & 53.35 & 51.00 & 70.00 & 64.23 & 54.90 & 59.66 \\
\hline SK-7 × APS-4 & 51.83 & 62.27 & 40.86 & 41.38 & 45.00 & 40.00 & 47.21 & 45.10 & 46.71 \\
\hline SK-7 × SK-6 & 50.72 & 57.94 & 40.86 & 60.13 & 61.00 & 40.00 & 36.26 & 40.20 & 48.39 \\
\hline SK-7 × Pam-117 & 42.81 & 43.79 & 37.71 & 56.79 & 59.00 & 50.00 & 44.42 & 45.10 & 47.45 \\
\hline FC-1 & 53.08 & 50.39 & 56.62 & 52.45 & 51.00 & 65.00 & 60.68 & 50.00 & 54.90 \\
\hline
\end{tabular}


Table.7 Rearing Performance of the oval foundation crosses during summer, 2018

\begin{tabular}{|c|c|c|c|c|c|c|c|c|}
\hline \multirow[t]{2}{*}{ Parental breeds } & \multirow{2}{*}{$\begin{array}{l}\text { Fecundity } \\
\text { (No.) }\end{array}$} & \multirow{2}{*}{$\begin{array}{c}\text { Hatching } \\
(\%)\end{array}$} & \multicolumn{2}{|c|}{ Yield / 10, 000 larvae brushed } & \multirow{2}{*}{$\begin{array}{l}\text { Single cocoon } \\
\text { wt. (g) }\end{array}$} & \multirow{2}{*}{$\begin{array}{l}\text { Single shell } \\
\text { wt.(g) }\end{array}$} & \multirow{2}{*}{$\begin{array}{c}\text { Shell ratio } \\
(\%)\end{array}$} & \multirow{2}{*}{$\begin{array}{c}\text { Pupation rate } \\
(\%)\end{array}$} \\
\hline & & & By No. & By Wt.(kg) & & & & \\
\hline CSR-27 × CSR -50 & 540 & 94.73 & 9600 & 14.71 & 1.72 & 0.36 & 20.94 & 91.50 \\
\hline CSR-27 $\times$ Pam-114 & 546 & 96.55 & 9660 & 14.48 & 1.69 & 0.35 & 20.47 & 92.50 \\
\hline CSR $-27 \times$ APS -5 & 499 & 93.00 & 9500 & 14.73 & 1.74 & 0.34 & 19.55 & 93.50 \\
\hline CSR-50 $\times$ CSR -27 & 518 & 92.73 & 9640 & 14.20 & 1.66 & 0.33 & 19.88 & 94.00 \\
\hline CSR-50 $\times$ Pam-114 & 561 & 95.24 & 9580 & 15.06 & 1.76 & 0.36 & 20.47 & 93.50 \\
\hline CSR $-50 \times$ APS -5 & 514 & 93.07 & 9700 & 14.74 & 1.71 & 0.34 & 19.65 & 93.00 \\
\hline Pam-114 × CSR-27 & 571 & 95.69 & 9620 & 15.37 & 1.79 & 0.37 & 20.73 & 92.00 \\
\hline Pam-114 × CSR-50 & 569 & 94.31 & 9620 & 15.57 & 1.81 & 0.38 & 20.78 & 91.50 \\
\hline Pam-114 × APS-5 & 539 & 92.96 & 9620 & 13.88 & 1.63 & 0.33 & 19.94 & 93.50 \\
\hline APS $-5 \times$ CSR -27 & 519 & 93.07 & 9660 & 13.37 & 1.57 & 0.30 & 19.10 & 94.00 \\
\hline APS- $5 \times$ CSR -50 & 537 & 93.21 & 9660 & 14.00 & 1.64 & 0.32 & 19.26 & 93.50 \\
\hline APS-5 × Pam-114 & 579 & 95.21 & 9580 & 14.82 & 1.74 & 0.36 & 20.75 & 94.00 \\
\hline $\mathrm{FC} 2$ & 578 & 94.34 & 9640 & 14.25 & 1.67 & 0.35 & 21.03 & 94.00 \\
\hline Average & 544 & 94.16 & 9622 & 14.55 & 1.70 & 0.34 & 20.20 & 93.12 \\
\hline $\mathrm{SD}$ & 26.33 & 1.25 & 49.97 & 0.61 & 0.07 & 0.02 & 0.66 & 0.94 \\
\hline
\end{tabular}

Table. 8 Evaluation index values of the oval foundation crosses during summer, 2018

\begin{tabular}{|c|c|c|c|c|c|c|c|c|c|}
\hline \multirow[t]{2}{*}{ Parental breeds } & \multirow{2}{*}{$\begin{array}{l}\text { Fecundity } \\
\text { (No.) }\end{array}$} & \multirow{2}{*}{$\begin{array}{l}\text { Hatching } \\
(\%)\end{array}$} & \multicolumn{2}{|c|}{ Yield / 10, 000 larvae brushed } & \multirow{2}{*}{$\begin{array}{l}\text { Single cocoon } \\
\text { wt. (g) }\end{array}$} & \multirow{2}{*}{$\begin{array}{l}\text { Single shell } \\
\text { wt.(g) }\end{array}$} & \multirow{2}{*}{$\begin{array}{c}\text { Shell ratio } \\
(\%)\end{array}$} & \multirow{2}{*}{$\begin{array}{l}\text { Pupation rate } \\
\text { (\%) }\end{array}$} & \multirow{2}{*}{$\begin{array}{c}\text { Average } \\
\text { E.I }\end{array}$} \\
\hline & & & By No & By Wt.(kg) & & & & & \\
\hline CSR-27 $\times$ CSR -50 & 48.44 & 54.56 & 45.60 & 52.58 & 52.86 & 60.00 & 61.14 & 34.04 & 51.15 \\
\hline CSR-27 × Pam-114 & 50.76 & 69.12 & 57.60 & 48.81 & 47.86 & 52.50 & 54.15 & 44.68 & 53.18 \\
\hline CSR-27 × APS-5 & 32.80 & 40.72 & 25.59 & 52.99 & 55.71 & 50.00 & 40.16 & 55.32 & 44.16 \\
\hline CSR-50 $\times$ CSR -27 & 40.28 & 38.56 & 53.60 & 44.31 & 44.29 & 45.00 & 45.16 & 60.64 & 46.48 \\
\hline CSR-50 × Pam-114 & 56.53 & 58.60 & 41.59 & 58.41 & 58.57 & 60.00 & 54.02 & 55.32 & 55.38 \\
\hline CSR-50 $\times$ APS-5 & 38.64 & 41.24 & 65.61 & 53.10 & 50.71 & 47.50 & 41.62 & 50.00 & 48.55 \\
\hline Pam-114 × CSR-27 & 60.33 & 62.20 & 49.60 & 63.41 & 62.14 & 65.00 & 57.96 & 39.36 & 57.50 \\
\hline Pam-114 × CSR-50 & 59.38 & 51.16 & 49.60 & 66.66 & 65.00 & 67.50 & 58.76 & 34.04 & 56.51 \\
\hline Pam-114 × APS-5 & 48.25 & 40.40 & 49.60 & 39.02 & 40.00 & 42.50 & 46.08 & 55.32 & 45.15 \\
\hline APS- $5 \times$ CSR -27 & 40.62 & 41.28 & 57.60 & 30.60 & 31.43 & 30.00 & 33.38 & 60.64 & 40.69 \\
\hline APS- $5 \times$ CSR-50 & 47.34 & 42.40 & 57.60 & 40.91 & 40.71 & 37.50 & 35.83 & 55.32 & 44.70 \\
\hline APS- $5 \times$ Pam-114 & 63.10 & 58.36 & 41.59 & 54.47 & 55.00 & 60.00 & 58.35 & 60.64 & 56.44 \\
\hline $\mathrm{FC} 2$ & 63.06 & 51.44 & 53.60 & 45.13 & 45.00 & 55.00 & 62.57 & 60.64 & 54.56 \\
\hline
\end{tabular}


Table.9 Rearing Performance of the constricted foundation crosses during autumn, 2018

\begin{tabular}{|c|c|c|c|c|c|c|c|c|}
\hline \multirow[t]{2}{*}{ Parental breeds } & \multirow{2}{*}{$\begin{array}{l}\text { Fecundity } \\
\text { (No.) }\end{array}$} & \multirow{2}{*}{$\begin{array}{l}\text { Hatching } \\
(\%)\end{array}$} & \multicolumn{2}{|c|}{ Yield / 10, 000 larvae brushed } & \multirow{2}{*}{$\begin{array}{c}\text { Single cocoon } \\
\text { wt. (g) }\end{array}$} & \multirow{2}{*}{$\begin{array}{l}\text { Single shell } \\
\text { wt.(g) }\end{array}$} & \multirow{2}{*}{$\begin{array}{c}\text { Shell ratio } \\
(\%)\end{array}$} & \multirow{2}{*}{$\begin{array}{c}\text { Pupation rate } \\
(\%)\end{array}$} \\
\hline & & & By No. & By Wt.(kg) & & & & \\
\hline APS-4 × SK-6 & 492 & 94.01 & 9490 & 13.03 & 1.69 & 0.32 & 18.63 & 92.00 \\
\hline APS-4 × Pam-117 & 518 & 95.61 & 9360 & 12.68 & 1.55 & 0.29 & 18.85 & 91.50 \\
\hline APS-4 × SK-7 & 492 & 93.21 & 9460 & 13.59 & 1.63 & 0.31 & 18.94 & 92.00 \\
\hline SK-6 $\times$ APS-4 & 480 & 95.03 & 9380 & 13.32 & 1.69 & 0.31 & 18.09 & 90.50 \\
\hline SK-6 $\times$ Pam-117 & 518 & 94.59 & 9480 & 12.12 & 1.47 & 0.25 & 16.85 & 91.50 \\
\hline SK-6 $\times$ SK-7 & 549 & 94.56 & 9640 & 14.15 & 1.54 & 0.32 & 20.44 & 93.00 \\
\hline Pam-117 × APS-4 & 525 & 93.63 & 9600 & 14.04 & 1.57 & 0.32 & 20.70 & 93.00 \\
\hline Pam-117 × SK-6 & 482 & 93.59 & 9480 & 12.72 & 1.53 & 0.30 & 19.75 & 92.50 \\
\hline Pam-117 × SK-7 & 544 & 95.88 & 9640 & 14.01 & 1.53 & 0.33 & 21.75 & 93.50 \\
\hline SK-7 $\times$ APS- 4 & 505 & 93.69 & 9420 & 13.24 & 1.70 & 0.32 & 18.94 & 91.50 \\
\hline SK-7 × SK-6 & 482 & 93.87 & 9520 & 13.54 & 1.61 & 0.31 & 19.01 & 92.50 \\
\hline SK-7 $\times$ Pam-117 & 537 & 96.77 & 9460 & 14.03 & 1.45 & 0.29 & 20.36 & 92.50 \\
\hline FC-1 & 502 & 93.56 & 9340 & 12.55 & 1.54 & 0.33 & 21.32 & 88.00 \\
\hline Average & 510 & 94.46 & 9482 & 13.31 & 1.58 & 0.31 & 19.51 & 91.85 \\
\hline SD & 24 & 1.08 & 98 & 0.66 & 0.08 & 0.02 & 1.37 & 1.41 \\
\hline
\end{tabular}

Table.10 Evaluation index values of the constricted foundation crosses during autumn, 2018

\begin{tabular}{|c|c|c|c|c|c|c|c|c|c|}
\hline \multirow[t]{2}{*}{ Parental breeds } & \multirow{2}{*}{$\begin{array}{l}\text { Fecundity } \\
\text { (No.) }\end{array}$} & \multirow{2}{*}{$\begin{array}{l}\text { Hatching } \\
(\%)\end{array}$} & \multicolumn{2}{|c|}{ Yield / 10, 000 larvae brushed } & \multirow{2}{*}{$\begin{array}{l}\text { Single cocoon } \\
\text { wt. (g) }\end{array}$} & \multirow{2}{*}{$\begin{array}{c}\text { Single shell } \\
\text { wt.(g) }\end{array}$} & \multirow{2}{*}{$\begin{array}{c}\text { Shell ratio } \\
(\%)\end{array}$} & \multirow{2}{*}{$\begin{array}{c}\text { Pupation rate } \\
(\%)\end{array}$} & \multirow{2}{*}{$\begin{array}{c}\text { Average } \\
\text { E.I }\end{array}$} \\
\hline & & & By No. & By Wt.(kg) & & & & & \\
\hline APS-4 $\times$ SK-6 & 42.50 & 45.79 & 50.82 & 45.76 & 63.81 & 52.50 & 43.60 & 51.06 & 49.48 \\
\hline APS-4 × Pam-117 & 53.38 & 60.60 & 37.55 & 40.40 & 45.81 & 40.75 & 45.18 & 47.52 & 46.40 \\
\hline APS-4 × SK-7 & 42.60 & 38.38 & 47.76 & 54.17 & 55.81 & 49.00 & 45.81 & 51.06 & 48.07 \\
\hline SK-6 $\times$ APS-4 & 37.29 & 55.28 & 39.59 & 50.12 & 63.25 & 47.50 & 39.64 & 40.43 & 46.64 \\
\hline SK-6 $\times$ Pam-117 & 53.46 & 51.20 & 49.80 & 32.04 & 36.13 & 18.75 & 30.57 & 47.52 & 39.93 \\
\hline SK-6 $\times$ SK-7 & 66.42 & 50.88 & 66.12 & 62.73 & 45.13 & 52.50 & 56.80 & 58.16 & 57.34 \\
\hline Pam-117 × APS-4 & 56.35 & 42.31 & 62.04 & 61.12 & 48.44 & 57.25 & 58.70 & 58.16 & 55.55 \\
\hline Pam-117 × SK-6 & 38.42 & 41.90 & 49.80 & 41.08 & 44.00 & 46.25 & 51.72 & 54.61 & 45.97 \\
\hline Pam-117 × SK-7 & 64.33 & 63.10 & 66.12 & 60.61 & 43.31 & 61.00 & 66.34 & 61.70 & 60.82 \\
\hline SK-7 × APS-4 & 48.04 & 42.87 & 43.67 & 48.91 & 65.13 & 56.13 & 45.87 & 47.52 & 49.77 \\
\hline SK-7 × SK-6 & 38.42 & 44.54 & 53.88 & 53.45 & 53.88 & 48.13 & 46.35 & 54.61 & 49.16 \\
\hline SK-7 $\times$ Pam-117 & 61.04 & 71.39 & 47.76 & 60.91 & 33.13 & 42.13 & 56.23 & 54.61 & 53.40 \\
\hline FC-1 & 46.83 & 41.67 & 35.51 & 38.53 & 44.50 & 58.75 & 63.22 & 22.70 & 43.96 \\
\hline
\end{tabular}


Table.11 Rearing Performance of the oval foundation crosses during autumn, 2018

\begin{tabular}{|c|c|c|c|c|c|c|c|c|}
\hline \multirow[t]{2}{*}{ Parental breeds } & \multirow{2}{*}{$\begin{array}{l}\text { Fecundity } \\
\text { (No.) }\end{array}$} & \multirow{2}{*}{$\begin{array}{c}\text { Hatching } \\
(\%)\end{array}$} & \multicolumn{2}{|c|}{ Yield / 10, 000 larvae brushed } & \multirow{2}{*}{$\begin{array}{l}\text { Single cocoon } \\
\text { wt. (g) }\end{array}$} & \multirow{2}{*}{$\begin{array}{l}\text { Single shell } \\
\text { wt.(g) }\end{array}$} & \multirow{2}{*}{$\begin{array}{c}\text { Shell ratio } \\
(\%)\end{array}$} & \multirow{2}{*}{$\begin{array}{c}\text { Pupation rate } \\
(\%)\end{array}$} \\
\hline & & & By No. & By Wt.(kg) & & & & \\
\hline CSR-27 × CSR -50 & 516 & 92.41 & 9640 & 13.02 & 1.58 & 0.31 & 19.33 & 93.50 \\
\hline CSR-27 $\times$ Pam-114 & 532 & 94.89 & 9720 & 14.16 & 1.47 & 0.31 & 20.92 & 94.00 \\
\hline CSR $-27 \times$ APS -5 & 493 & 93.61 & 9560 & 13.05 & 1.55 & 0.31 & 19.83 & 93.00 \\
\hline CSR-50 $\times$ CSR -27 & 490 & 94.88 & 9480 & 13.00 & 1.56 & 0.31 & 19.91 & 92.00 \\
\hline CSR-50 $\times$ Pam-114 & 526 & 94.44 & 9720 & 14.92 & 1.72 & 0.38 & 22.06 & 93.50 \\
\hline CSR $-50 \times$ APS -5 & 510 & 94.69 & 9540 & 13.78 & 1.63 & 0.34 & 21.06 & 92.50 \\
\hline Pam-114 × CSR-27 & 533 & 96.88 & 9780 & 13.65 & 1.54 & 0.35 & 22.81 & 95.00 \\
\hline Pam-114 × CSR-50 & 513 & 94.32 & 9660 & 13.78 & 1.57 & 0.33 & 21.33 & 94.50 \\
\hline Pam-114 × APS-5 & 499 & 93.85 & 9340 & 11.91 & 1.47 & 0.28 & 19.26 & 90.50 \\
\hline APS $-5 \times$ CSR -27 & 500 & 94.33 & 9300 & 11.72 & 1.45 & 0.29 & 19.67 & 91.50 \\
\hline APS- $5 \times$ CSR-50 & 531 & 94.89 & 9600 & 13.71 & 1.60 & 0.34 & 21.09 & 92.50 \\
\hline APS-5 × Pam-114 & 499 & 94.57 & 9660 & 12.71 & 1.50 & 0.30 & 19.68 & 94.00 \\
\hline $\mathrm{FC} 2$ & 543 & 96.80 & 9620 & 13.81 & 1.53 & 0.32 & 21.13 & 92.50 \\
\hline Average & 514 & 94.66 & 9586 & 13.32 & 1.55 & 0.32 & 20.62 & 93.00 \\
\hline $\mathrm{SD}$ & 17 & 1.18 & 143 & 0.89 & 0.07 & 0.03 & 1.10 & 1.26 \\
\hline
\end{tabular}

Table.12 Evaluation index values of the oval foundation crosses during autumn, 2018

\begin{tabular}{|c|c|c|c|c|c|c|c|c|c|}
\hline \multirow[t]{2}{*}{ Parental breeds } & \multirow{2}{*}{$\begin{array}{l}\text { Fecundity } \\
\text { (No.) }\end{array}$} & \multirow{2}{*}{$\begin{array}{l}\text { Hatching } \\
(\%)\end{array}$} & \multicolumn{2}{|c|}{ Yield / 10, 000 larvae brushed } & \multirow{2}{*}{$\begin{array}{l}\text { Single cocoon } \\
\text { wt. (g) }\end{array}$} & \multirow{2}{*}{$\begin{array}{l}\text { Single shell } \\
\text { wt.(g) }\end{array}$} & \multirow{2}{*}{$\begin{array}{c}\text { Shell ratio } \\
(\%)\end{array}$} & \multirow{2}{*}{$\begin{array}{l}\text { Pupation rate } \\
\text { (\%) }\end{array}$} & \multirow{2}{*}{$\begin{array}{c}\text { Average } \\
\text { E.I }\end{array}$} \\
\hline & & & By No & By Wt.(kg) & & & & & \\
\hline CSR-27 $\times$ CSR -50 & 51.18 & 30.89 & 53.78 & 46.59 & 53.93 & 45.00 & 38.31 & 53.97 & 46.71 \\
\hline CSR-27 × Pam-114 & 60.35 & 51.91 & 59.37 & 59.44 & 38.86 & 46.00 & 52.76 & 57.94 & 53.33 \\
\hline CSR-27 × APS-5 & 37.59 & 41.10 & 48.18 & 47.00 & 50.50 & 46.00 & 42.78 & 50.00 & 45.39 \\
\hline CSR-50 $\times$ CSR -27 & 36.06 & 51.82 & 42.59 & 46.36 & 51.58 & 46.92 & 43.51 & 42.06 & 45.11 \\
\hline CSR-50 × Pam-114 & 57.00 & 48.14 & 59.37 & 68.01 & 74.36 & 69.83 & 63.07 & 53.97 & 61.72 \\
\hline CSR-50 × APS-5 & 47.82 & 50.25 & 46.78 & 55.11 & 61.79 & 57.92 & 53.97 & 46.03 & 52.46 \\
\hline Pam-114 × CSR-27 & 61.37 & 68.81 & 63.57 & 53.76 & 49.07 & 60.67 & 69.87 & 65.87 & 61.62 \\
\hline Pam-114 × CSR-50 & 49.12 & 47.12 & 55.17 & 55.15 & 52.57 & 54.83 & 56.48 & 61.90 & 54.04 \\
\hline Pam-114 × APS-5 & 41.00 & 43.14 & 32.80 & 34.10 & 38.14 & 37.50 & 37.61 & 30.16 & 36.81 \\
\hline APS- $5 \times$ CSR -27 & 41.76 & 47.20 & 30.00 & 31.97 & 36.14 & 38.58 & 41.33 & 38.10 & 38.14 \\
\hline APS- $5 \times$ CSR-50 & 60.24 & 51.95 & 50.98 & 54.36 & 57.71 & 56.08 & 54.25 & 46.03 & 53.95 \\
\hline APS- $5 \times$ Pam-114 & 41.00 & 49.24 & 55.17 & 43.10 & 43.07 & 41.83 & 41.46 & 57.94 & 46.60 \\
\hline $\mathrm{FC} 2$ & 67.12 & 68.14 & 52.38 & 55.55 & 46.57 & 50.83 & 54.67 & 46.03 & 55.16 \\
\hline
\end{tabular}


In conclusion, three constricted foundation crosses SK-6 $\times$ SK-7, Pam-117 $\times$ APS-4 and Pam-117 $\times$ SK-7 and four oval foundation crosses CSR-27 $\times$ Pam-114, CSR-50 $\times$ Pam114, Pam-114 $\times$ CSR-27 and Pam-114 $\times$ CSR-50 were found suitable for temperate region of Jammu \& Kashmir which will be utilised after further evaluation for the development of bivoltine silkworm double hybrid/ hybrids suitable to temperate region of Jammu and Kashmir as an alternate for ruling CSR double hybrid $(\mathrm{FC} 2 \times \mathrm{FC} 1)$.

\section{Acknowledgments}

Authors wish to express a deep sense of gratitude to Central Silk Board and Central Sericultural Research and Training Institute, Pampore, Jammu and Kashmir for providing financial assistance and laboratory facilities to carry out the research work.

\section{References}

Basavaraja, H. K., Nirmal Kumar, S., Suresh Kumar, N., Mal Reddy, N., Kshama Giridhar,

Ahshan, M. M. and Datta, R. K. (1995). New productive bivoltine hybrids. Indian Silk, 34: 5-9.

Basavaraja, H. K, N. Suresh Kumar and S. B. Dandin (2006). Breeding strategies for

productivity and quality in Mulberry Silkworm Breeders' Meet, 14th-15th Feb 2006, CSRTI, Berhamporepp.1-6.

Bharath Kumar Neelaboina., Gulzar Ahmad Khan, Mudasir Gani, Shakeel Ahmad, Mir Nisar

Ahmad and M.K. Ghosh (2018a). Exploration of sericulture in unexplored region of Jammu and Kashmir. Journal of Entomolozy and Zoolozy Studies, 6(4): 1922-1925.

Bharath Kumar, Neelaboina., Shivkumar, Gani, M., Babulal and Ghosh, M. K. (2017). Assessment of performance of autumn crop over spring in temperate region of Jammu \& Kashmir. Journal of Agroecology and Natural Resource Management, 4 (2): 112 - 114.

Bharath Kumar Neelaboina., Shivkumar, Gulzar Ahmad khan, Mudasir Gani, Mir Nisar

Ahmad and M.K. Ghosh (2018b). Impact of Climate Change on Agriculture and allied sectors. Journal of Entomolozy and Zoolozy Studies, 6(5): 426-429

Farooq M, Singh TP, Nooruddin, Rufaie Z. H., Baqual M., Dar H. U. (2006) Second commercial crop to make sericulture a more profitable in Kashmir. Proceedings of regional Seminar on Prospects and Problems of Sericulture as are economic enterprise in North West India. 275-276.

Krishnaswami, S. (1978). New technology of silkworm rearing, Bulletin No. 2, CSR\&TI, Mysore, India, pp. 1-24.

Malik MA, Kamili AS, Sofi AM, Malik GN, Sabahat A, Bhat SA. (2009)Second commercial silkworm rearing in Kashmir - A ray of hope. Indian Silk, 9:10-11.

Mano, Y., Nirmalkumar, S., Basavaraja, H.K., Mal Reddy, N. and Datta, R.K. (1993). A new method to select promising silkworm breed/hybrid combinations. Indian Silk, 31(10): 53.

Miyashita, V. (1986) A report on mulberry and training methods suitable to bivoltine rearing in Karnataka. Central Silk Board, pp.1-7.

Moorthy SM, K. Mandal, N. B. Kar and S. K. Das. (2011). Identification of suitable bivoltine foundation cross for sustainable bivoltine silkworm seed crop in tropics. The Bioscan,6(4):697700.

Nisar, M., Chisti, M.Z. and Khan, M.A. (2013). Studies on the identification of summer specific silkworm Bombyx mori L. hybrids under temperate climatic 
conditions of Jammu and Kashmir, India. J. Intl. Acad. Res. Multidisci, 1(3): 1-14.

Quadir, S. M., Nisar, M., Khan, M. A. and Ahsan, M. M. (2000). Identification of season specific silkworm hybrids for temperate climatic conditions of Kashmir. In: National Conference on Strategies for Sericulture Research and Development, Central Sericultural Research and Training Institute, Srirampura, Mysore, India, 16-18, November 2000, pp.21.

Sahaf KA, Bhat SA, Mir Nisar A. (2016). Sericulture in North-west India with special reference to temperate regionproblems and prospects. National seminar on sericulture development in temperate region- problems and prospects. 34-38.

Shabir Ahmad Bhat, Ravi Kant, Naveena Nazim, Mohd Rafiq Bhat, Noor-ul-Din,
Munazah.

Yaqoob and Nageena Nazir (2018). Genetic variability analysis of some bivoltine silkworm (Bombyx mori L.) breeds. Journal of Pharmacognosy and Phytochemistry, 7(3): 889-892.

Suresh Kumar, N., Basavaraja, H.K., Joge, P.G,, Mal Reddy, N., Kalpana, G.V. and Dandin, S.B. (2006). Development of new robust bivoltine hybrid (CSR46 $\mathrm{x}$ CSR47) of Bombyx mori L. for the tropics. Indian J. Seric, 45(1): 21-29.

Trag AR, Kamili AS, Malik GN, Kukiloo FA. (1992). Evolution of high yielding bivoltine silkworm, Bombyx mori genotypes. Sericologia. 32:321-324.

Tsukada, M., Islam, S., Arai, T., Bosch, A and Fred, G. (2005). Microwave irradiation technique to enhance protein fiber properties. Autex Res J, 5(1): 40-8.

\section{How to cite this article:}

Bharath Kumar Neelaboina, Shivkumar, Mir Nisar Ahmad and Ghosh, M.K. 2019. Evaluation of Elite Bivoltine Silkworm (Bombyx mori L.) Foundation Crosses Suitable for Temperate Region of Jammu \& Kashmir. Int.J.Curr.Microbiol.App.Sci. 8(01): 2980-2990. doi: https://doi.org/10.20546/ijcmas.2019.801.317 